\section{Dinucleotide repeat polymorphism in human GLUT2/liver facilitative glucose transporter gene on chromosome 3}

\author{
M.Granqvist, K.Xiang, M.Seino, H.Fukumoto and \\ G.I.Bell* \\ Howard Hughes Medical Institute, University of Chicago, \\ 5841 S. Maryland Avenue, Box 391, Chicago, IL 60637, \\ USA
}

Primers/Description: Two primers (GLUT2-1, 5'-TCCGTCAGCAGCTATTCTAG-3' and GLUT2 - 2, 5'-CAAATAGTCCTCATGCAGAA-3') were used to amplify a 184-222 bp CA and TA repeat-rich region in intron 1 of the human GLUT2 gene.

Frequency: Fourteen alleles were observed in 68 unrelated Caucasians. The heterozygosity was $91 \%$.

\begin{tabular}{llllll}
\multicolumn{2}{l}{ Allele bp } & Frequency & \multicolumn{2}{l}{ Allele bp } & Frequency \\
L1 & 222 & 0.02 & L2 & 220 & 0.02 \\
L3 & 218 & 0.06 & L4 & 216 & 0.02 \\
L5 & 214 & 0.06 & L6 & 212 & 0.01 \\
L7 & 202 & 0.01 & L8 & 200 & 0.11 \\
L9 & 198 & 0.18 & L10 & 196 & 0.31 \\
L11 & 194 & 0.05 & L12 & 192 & 0.05 \\
L13 & 186 & 0.04 & L14 & 184 & 0.06
\end{tabular}

Chromosomal Localization: GLUT2 was assigned to chromosome 3q26.1-q26.3 (1).

Mendelian Inheritance: Co-dominant inheritance was observed in five nuclear families.

Other Comments: The PCR was preformed using ${ }^{32} \mathrm{P}$-labeled GLUT2 -1 and unlabeled GLUT2 -2 for 30 cycles; denaturation at $94^{\circ} \mathrm{C}$ for $1 \mathrm{~min}$; annealing at $55^{\circ} \mathrm{C}$ for $1 \mathrm{~min}$ and extension at $72^{\circ} \mathrm{C}$ for $2 \mathrm{~min}$. The PCR products were analyzed on a $5 \%$ denaturing polyacrylamide gel (Figure 1). The dinucleotide repeat sequence in intron 1 was of the form $(\mathrm{TA})_{16}(\mathrm{CA})_{6}(\mathrm{TA})_{5}(\mathrm{CA})_{9}$; the complete sequence of this region is available from the authors.

References: 1) Fukumoto,H. et al. (1988) Proc. Natl. Acad. Sci. USA 85, 5434.

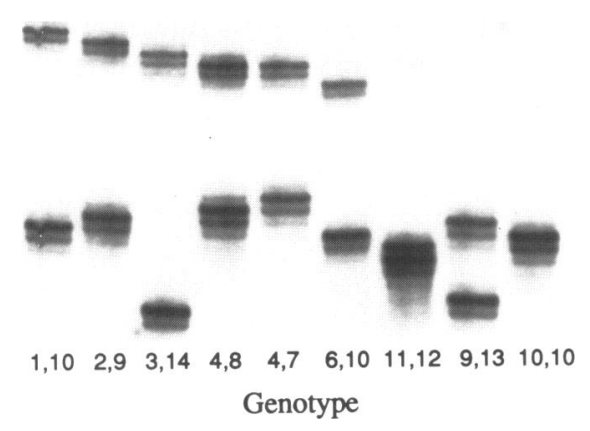

Figure 1. PCR amplification of dinucleotide repeat DNA polymorphism in human GLUT2 gene. The genotypes are noted at the bottom of the figure.

* To whom correspondence should be addressed

\section{Pentanucleotide repeat length polymorphism at the human CD4 locus}

\author{
M.C.Edwards, P.R.Clemens, M.Tristan, A.Pizzuti and \\ R.A.Gibbs \\ Institute for Molecular Genetics, Baylor College of \\ Medicine, Houston, TX 77030, USA
}

Source and Description of Clone: Partial sequencing of an approximately $15 \mathrm{~kb}$ lambda insert (lambda hgl) containing exons $1-3$ of the human CD4 gene revealed a tract of 12 CTTTT's. The sequences flanking the pentanucleotide repeat (CTTTT)n were then used to design PCR primers that amplified a 113 base pair fragment from lambda hg1.

\section{Primer Sequences:}

5'-TTGGAGTCGCAAGCTGAACTAGC-3'(CTTTT strand), 5'-GCCTGAGTGACAGAGTGAGAACC-3'(AAAAG strand)

Polymorphism: Allelic fragments in 5 bp intervals from 88 to 128 bp.

Frequencies: (a) in 100 unrelated Caucasians, (b) in 14 unrelated North American Blacks:

$\begin{array}{llllllll}\text { Allele (bp) } & \text { Freq.(a) } & \text { Freq.(b) } & \text { Allele (bp) } & \text { Freq.(a) } & \text { Freq.(b) } \\ \text { A8 } & 88 & 0.365 & 0.286 & \text { A4 } 108 & 0.300 & 0.036 \\ \text { A7 } & 93 & 0.295 & 0.214 & \text { A3 } 113 & 0.025 & 0.250 \\ \text { A6 } & 98 & 0.005 & 0.107 & \text { A2 } & 118 & 0.005 & 0.036 \\ \text { A5 } & 103 & 0.005 & 0.036 & \text { A1 } 128 & 0.000 & 0.036\end{array}$

Mendelian Inheritance: Mendelian inheritance was observed in 4 three-generation families and 2 two-generation families.

Chromosomal Location: The human CD4 gene has been mapped to the short arm of chromosome 12 (1).

PCR Conditions: PCR was performed on 50 ng genomic DNA using $25 \mathrm{pmol}$ of each primer. Thermocycling conditions were $94^{\circ} \mathrm{C} 5$ mins then $94^{\circ} \mathrm{C} 30 \mathrm{secs}, 55^{\circ} \mathrm{C} 30$ secs and $68^{\circ} \mathrm{C}, 2 \mathrm{~min}$. for 25 cycles in reaction conditions described (2).

Acknowledgements: Thanks to Dan Littman for supplying lambda clones containing the CD4 gene. Research supported in part by grants USPH U01 AI30243 and RR06404.

References: 1) Isobe,M. et al. (1986) Proc. Natl. Acad. Sci. USA 83, 4399-4402. 2) Gibbs,R.A. et al. (1990) Genomics 7, $235-244$.

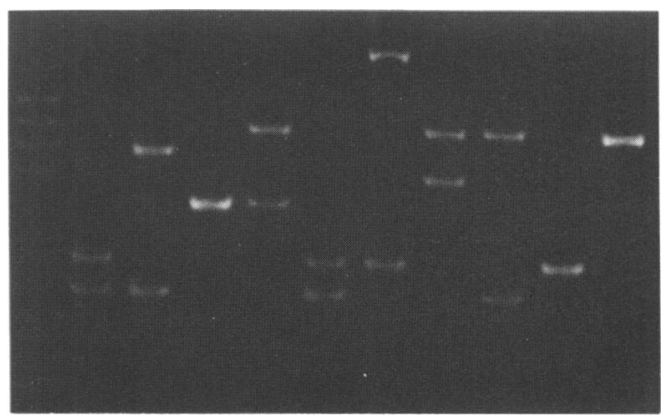

Figure. Alleles were detected by electrophoresis on $10 \%$ native acrylamide gels followed by ethidium bromide staining. The first lane shows size standards generated by a mixture of known alleles; other lanes show the patterns generated from individual DNA samples. 